\title{
Unexpected D-type Interlopers in the Inner Main Belt
}

\author{
Francesca E. DeMeo ${ }^{\mathrm{a}, \mathrm{b}, \mathrm{c}}$, Richard P. Binzel ${ }^{\mathrm{b}}$, Benoît Carry ${ }^{\mathrm{d}, \mathrm{e}}$, David Polishook $^{\mathrm{b}}$, Nicholas A. Moskovitz $^{\mathrm{b}}$ \\ ${ }^{a}$ Harvard-Smithsonian Center for Astrophysics, 60 Garden Street, MS-16, Cambridge, MA, 02138, USA.fdemeo@cfa.harvard.edu \\ ${ }^{b}$ Department of Earth, Atmospheric, and Planetary Sciences, Massachusetts Institute of Technology, 77 Massachusetts Avenue, Cambridge, MA 02139 USA \\ ${ }^{c}$ Hubble Fellow \\ dInstitut de Mécanique Céleste et de Calcul des Éphémérides, Observatoire de Paris, UMR8028 CNRS, 77 av. Denfert-Rochereau 75014 Paris, France \\ ${ }^{e}$ European Space Astronomy Centre, ESA, P.O. Box 78, 28691 Villanueva de la Cañada, Madrid, Spain
}

\begin{abstract}
Very red featureless asteroids (spectroscopic D-types) are expected to have formed in the outer solar system far from the sun. They comprise the majority of asteroids in the Jupiter Trojan population, and are also commonly found in the outer main belt and among Hildas. The first evidence for D-types in the inner and middle parts of the main belt was seen in the Sloan Digital Sky Survey (SDSS). Here we report follow-up observations of SDSS D-type candidates in the near-infrared. Based on follow up observations of 13 SDSS D-type candidates, we find a $\sim 20 \%$ positive confirmation rate. Known inner belt D-types range in diameter from roughly 7 to 30 kilometers. Based on these detections we estimate there are $\sim 100$ inner belt D-types with diameters between 2.5 and $20 \mathrm{~km}$. The lower and upper limits for total mass of inner belt D-types is $2 \times 10^{16} \mathrm{~kg}$ to $2 \times 10^{17} \mathrm{~kg}$ which represents $0.01 \%$ to $0.1 \%$ of the mass of the inner belt. The inner belt D-types have albedos at or above the upper end typical for D-types which raises the question as to whether these inner belt bodies represent only a subset of D-types, they have been altered by external factors such as weathering processes, or if they are compositionally distinct from other D-types. All D-types and candidates have diameters less than $30 \mathrm{~km}$, yet there is no obvious parent body in the inner belt. Dynamical models have yet to show how D-types originating from the outer solar system could penetrate into the inner reaches of the Main Belt under current scenarios of planet formation and subsequent Yarkovsky drift.
\end{abstract}

\section{Keywords: ASTEROIDS, SPECTROSCOPY}

\section{Introduction}

Spectral D-type asteroids are defined by their very red spectral slopes and typical lack of distinguishing absorption features in the visible and near-infrared wavelength ranges (Tholen 1984; Bus and Binzel 2002a; DeMeo et al. 2009a). Despite a lack of clear absorption bands to determine their composition, D-types are expected to be rich in organic compounds (Gradie and Veverka 1980, Cruikshank and Kerridge 1992). Spectral surveys of D-types include Lagerkvist et al. (1993), Fitzsimmons et al. (1994), Fornasier et al. (2004), Dotto et al. (2006), Fornasier et al. (2007), Roig et al. (2008b), Emery et al. (2011), and Yang and Jewitt (2011).

Traditionally, D-types have only been found in the outer main belt, beyond 2.82 AU into the Jovian Trojan population at 5.2 AU. They are the dominant spectral type among Trojan asteroids and make up an important fraction of Hildas, Cybeles, and outer main-belt asteroids (Gradie and Tedesco 1982, Grav et al. 2012; DeMeo and Carry 2013). Their spectral slopes are significantly higher than for $\mathrm{C}$, and X-types found in the main asteroid belt (Tholen 1984; Bus and Binzel 2002b; DeMeo et al.2009a), but lower (at visible wavelengths) than many of the ultra-red Centaurs and TNOs such as (83982) Crantor, (42301) $2001 \mathrm{UR}_{163}$, and the prototypical (5145) Pholus (Alvarez-Candal et al.2008, Fornasier et al.2009; DeMeo et al. 2009b; Perna et al. 2010, Cruikshank et al. 1998).
A few D-types have been found among the NEO population (Binzel et al. 2004, DeMeo and Binzel 2008), but they have been conspicuously absent from the inner and middle asteroid belt regions ranging from 2.0 to 2.82 AU (Bus 1999; Bus and Binzel 2002b). This absence has fallen in line with dynamical models that suggest D-type asteroids formed farther out in the solar system and were transported inward by planetary migration but made it only as far as the outer main belt (Levison et al.2009, Morbidelli et al. 2005). The inner, middle, and outer sections of the main belt, ranging from 2.0-2.5, 2.5-2.82, and 2.82-3.2 AU respectively, are separated by the $3: 1$ and 5:2 mean motion resonances.

Evidence for a small number of D-type asteroids in the inner and middle asteroid belt has been seen in the Sloan Digital Sky Survey (SDSS, Ivezić et al. 2001, 2002) by Carvano et al. (2010) and DeMeo and Carry (2013). In this work we followup these "SDSS D-type candidates" with near-infrared spectral measurements and confirm the presence of D-types in the inner belt. We present the spectra, determine the positive detection rate of D-type asteroids based on SDSS candidates, calculate the total expected D-types in the inner belt according to their size distribution and mass, and discuss potential dynamical implications. 


\section{Observations}

\subsection{Target Selection}

Candidate D-types were chosen among objects observed in the Sloan Digital Sky Survey (SDSS) Moving Object Catalog (MOC). We use the fourth release (MOC4), including observations prior to March 2007. We restrict the sample based on data quality. Detail of the sample selection and classification can be found in DeMeo and Carry (2013). From this subset we create a list of all objects in the inner belt that have been classified at least once as a D-type. We then shorten this list further by removing any objects that have more than one observation that differs significantly from a D-type, such as an object that is classified once as an S and once as a D. Our final list includes 79 inner belt SDSS candidate D-types, 11 of which have been observed multiple times (Table 1). We also list the three Hungaria and three Phocaea candidates. We observed 13 targets using SpeX at the IRTF and FIRE at Magellan.

\section{[Table 1 about here.]}

\subsection{SpeX Observations}

Observations were taken on the 3-meter NASA Infrared Telescope Facility at the Mauna Kea Observatory. We use the instrument SpeX (Rayner et al. 2003), a near-infrared spectrograph in low resolution mode over 0.8 to $2.5 \mu \mathrm{m}$.

Objects are observed near the meridian (usually $<1.3$ airmass) in two different positions (typically denoted A and B) on a $0.8 \times 15$ arcsecond slit aligned north-south. Exposure times are typically 120 seconds, and we measure 8 to $12 \mathrm{~A}-\mathrm{B}$ pairs for each object. Solar analog stars are observed at similar airmass throughout the night. We use the same set of solar analogs as the SMASS program (Binzel et al. 2004, 2006) that have been in use for over a decade. Uncertainties in spectral slope on the IRTF using these consistent set of stars at low airmass is estimated to be around $5 \%$ of the measured slope value. Observations were taken in good weather conditions and observations of other objects throughout the night provide confidence that there were no major systematic slope issues.

Reduction and extraction is performed using the Image Reduction and Analysis Facility (IRAF) provided by the National Optical Astronomy Observatories (NOAO) (Tody 1993). Correction in regions with strong telluric absorption is performed in IDL using an atmospheric transmission (ATRAN) model by Lord (1992). The final spectrum for each object is created by dividing the telluric-corrected asteroid spectrum by the average of the telluric-corrected solar star spectra throughout that night. More detailed information on the observing and reduction procedures can be found in Rivkin et al. (2004) and DeMeo and Binzel (2008).

\subsection{FIRE Observations}

Observations were taken on the 6.5-meter Magellan Telescope at Las Campanas Observatory. We use the instrument Folded-port InfraRed Echellette (FIRE; Simcoe et al. 2013) in high-throughput, low-resolution prism mode with a slit width of 0.8 arcsecond oriented toward the parallactic angle. Exposures of 180 seconds were used for asteroids to avoid saturation due to thermal emission from the instrument and telescope at the long wavelength end (past $2.2 \mu \mathrm{m}$ ).

The readout mode sample-up-the-ramp was used for asteroid observations requiring exposure times in multiples of $10.7 \mathrm{sec}-$ onds. For stars readout mode Fowler 2 was used. Standard stars chosen were a combination of well-established solar analogs used for the past decade in our IRTF program and newly measured G2V stars that are dimmer and better suited for a larger, southern hemisphere telescope. Standard stars typically needed to be defocused to avoid saturation. Neon Argon lamp spectra were taken for wavelength calibration. Quartz lamp dome flats were taken for flat field corrections.

For FIRE data reduction, we used an IDL pipeline designed for the instrument based on the Spextool pipeline (Cushing et al. 2004). Among the pipeline settings, we use the boxcar extraction (boxcar=1) and local sky subtraction (nolocal=1). Tests of combinations of parameters show the best results with these settings. Because this slit is long (50"), for the sky correction, we use sky in the slit from the same exposure as the asteroid rather than $\mathrm{AB}$ pair subtractions. The observational circumstances are provided in Table 2

[Table 2 about here.]

\section{Results}

We confirmed 3 D-type inner belt asteroids from SDSS follow-up observations. Of SDSS candidates that had multiple observations 2 out of 5 were found to be D (40\% confirmation success). For singly observed SDSS candidates $1 / 7$ was confirmed (15\%). We note that asteroids (14291) and (125102) had spectral slopes that put them on the border of the X and D classes (if they were to be considered as D, the fraction confirmed would raise to $60 \%$ and $28 \%$ respectively). There is ambiguity between classes particularly for slopes near the boundaries of two classes. While formal classifications boundaries have been defined (e.g., Tholen 1984, Bus and Binzel 2002a, DeMeo et al. 2009a) these strict boundaries are in some cases artificial. Additionally, observational effects, such as airmass differences between object and solar analog, phase angle of the observations, and loss of light in the slit, cause spectral slope uncertainties on the order of $5 \%$ that could cause an object on the border to cross over to another class. For this reason we choose to include only the most distinctly D-type spectra where there is no ambiguity and thus do not consider the two border cases as D-types in order to be most conservative in assigning an asteroid to the D class. We thus provide a lower limit to the number of D-types in the inner belt.

We find all the confirmed D-types have $\mathrm{H}$ magnitudes less than 15 , while all 4 candidates with $H$ greater than 15 were false positives. Because of the small size of our sample, we do not know if this indicates fewer D-types at smaller sizes or is merely a reflection of a higher false positive rate for presumably lower SNR for data of the smaller, dimmer objects. 
Fig. 1 plots the spectra of confirmed inner belt D-type asteroids. The three SDSS candidates look strikingly similar. The spectra of (3282) Spencer Jones, (15112) $2000 \mathrm{EE}_{17}$, and (17906) $1999 \mathrm{FG}_{32}$ have slopes (between 0.85 and $2.45 \mu \mathrm{m}$ ) of $6.4,5.6$, and $5.9 \% / 1000 \AA$, respectively which is very comfortably within the D-type slope range of $\gtrsim 2.5 \% / 1000 \AA$ (DeMeo et al. 2009a).

The SDSS D-type candidates that were false positives are plotted in Fig. 2 and exhibit a wide range of spectral types. For many of these objects the false positives could be due to real slope difference between the visible and near-infrared. The asteroids in the right panel of Fig. 2 have distinct one- and twomicron absorption features indicating the presence of the minerals olivine and pyroxene, placing these spectra in the $\mathrm{S}, \mathrm{K}$, and $\mathrm{L}$ classes. We suspect that for these poorer matches, the false positive is due to the noise level of the SDSS measurement for that particular object and not due to a serious mismatch in the visible and near-infrared spectral characteristics.

Beyond the candidates in this survey, we find one asteroid with spectral measurements in both the visible and nearinfrared range that place it in the D class: (908) Buda (DeMeo et al.2009a). Asteroid (908) Buda, taken as part of the SMASS survey (Bus and Binzel 2002b; DeMeo et al. 2009a), was classified as an L-type by the visible wavelength survey, whereas the near-infrared data are inconsistent with an L-type, putting it in the D class. The orbital parameters of this object had not been reexamined after the classification change until now. This object has a moderately low to medium albedo of $0.087 \pm 0.007$ (Masiero et al.2011) from WISE and 0.16 \pm 0.02 (Tedesco et al. 2002) or $0.14 \pm 0.01$ (Ryan and Woodward 2010) from IRAS and a large spectral slope. The spectrum and two interesting features: a subtle absorption band centered near 0.9 microns and a distinct flattening of the spectrum to a more neutral slope past 2 microns. While the spectral slope clearly places (908) Buda in the D-class its moderate albedo would place it in the M class in the Tholen system. Similar subtle features on other asteroids are attributed to low iron, low calcium orthopyroxene (Ockert-Bell et al. 2008, Fornasier et al. 2010), although these objects fall in the Tholen M class or Bus-DeMeo Xk class. The visible wavelength survey by Lazzaro et al. (2004) detected 5 inner belt D-types, asteroids (565), (732), (1689), (2105) and (4103), the orbits of which had not been examined until now. Most of their albedos, however, are also significantly larger than typical for D-types: $0.11 \pm 0.02,0.14 \pm 0.02,0.18 \pm 0.05$, $0.16 \pm 0.03$, and $0.25 \pm 0.06$ (Masiero et al. 2011).

[Figure 1 about here.]

[Figure 2 about here.]

\section{Comparison with NEOs}

In the SMASS (Binzel et al. 2004, 2006) dataset of nearEarth objects (NEOs), there are 12 known D-types. While it had traditionally been assumed that these D-type NEOs come from the Jupiter Family Comet region or possibly the outer belt, source region probabilities derived from the model by Bottke et al. (2002) suggest that a significant number of these D-types (7/12) have orbits that make them highly likely to have originated in the middle or inner belt. All 7 of these NEOs have semi-major axes less then $2.5 \mathrm{AU}$, while those likely to have originated in the outer belt or from the Jupiter Family Comets have semi-major axes greater than $2.5 \mathrm{AU}$. Table 3 provides a probability estimate for an NEO's origin from multiple sources: a Jupiter Family Comet (JFC), outer asteroid belt (OB), 3:1 mean-motion resonance (3:1), Mars Crosser (MC), and the $v_{6}$ resonance (N6). While the relative abundance of D-types in the inner belt is much lower than for the outer regions, the $3: 1$ and $v_{6}$ resonance delivery mechanisms are significantly more efficient, meaning it could be possible that some of the NEO D-types actually originate from the inner belt.

[Table 3 about here.]

\section{Bias-corrected abundance and distribution}

We seek to use our observed sample to determine the total number of D-types expected to exist in the inner main belt. Here we exclude the Hungarias and Phocaeas because we do not have a large enough sample to determine the follow-up success rate in those regions. The SDSS survey is efficient to $\mathrm{H}$ magnitudes of about 17 in the inner belt (Ivezić et al. 2001; DeMeo and Carry 2013). We determine the expected total number of Dtypes in each $\mathrm{H}$ magnitude bin for the SDSS sample $\left(\mathrm{N}_{\mathrm{SDSS}, \mathrm{D}}\right)$ by first multiplying the number of SDSS D-type candidates by the success rate $(1 / 6$ or $2 / 5)$ based on the number of singly or multiply observed objects in each bin. Note we use $1 / 6$ because we exclude the one Hungaria asteroid that was observed. We then determine the number of inner belt objects ( $\left.\mathrm{N}_{\text {SDSS,total }}\right)$ from the entire SDSS sample over that size range in the inner belt and then calculate what fraction the D-types represent (D fraction). We can then apply that fraction to the total number of objects that exist $\left(\mathrm{N}_{\mathrm{MPC}}\right)$ to find the total number of inner belt D-types $\left(\mathrm{N}_{\mathrm{MPC}, \mathrm{D}}\right)$. The AstOrb database hosted at the Minor Planet Center (MPC) is essentially complete in the inner belt to $\mathrm{H}=16$ (DeMeo and Carry 2013). The MPC is $71 \%$ complete in the inner belt between $16<\mathrm{H}<17$, so we apply a correction factor to account for the expected undiscovered objects.

We place upper limits equal to the mean of the Poisson distribution for which there is a $90 \%$ chance of observing $n+1$ or more candidates where $\mathrm{n}$ is the number of expected objects in a given $\mathrm{H}$ bin of our SDSS sample (for $\mathrm{H}$ bins between $\mathrm{H}$ of 12 and $16 \mathrm{n}$ is $1,2,4,4,3$, respectively) as was done in similar work for V-types by Moskovitz et al. (2008). The lower limit is equal to the mean of the Poisson distribution for which there is a $90 \%$ chance of observing $n-1$ or fewer candidates. Table 4 provides the total number of D-types in the inner belt for each $\mathrm{H}$ magnitude bin.

Even though the SDSS survey is efficient to an $\mathrm{H}$ magnitude of 17, it is severely biased against asteroids with $\mathrm{H}<12$ because they saturated the detector during observations. The spectral surveys make up for this bias because they are nearly $100 \%$ complete down to $\mathrm{H}=12$ in the inner belt (DeMeo and Carry 
2013). Five D-types were identified in the inner belt from Lazzaro et al. based on visible wavelengths. Among the sample of asteroids observed at visible plus near-infrared wavelengths, all near-ir D-types have high slopes in the visible, but the reverse is not always true for D-types with only visible measurements (DeMeo et al.2009a). Additionally, the albedos for these targets are significantly higher than for typical D-types. For these reasons we do not include them in our calculations for this work, but if near-infrared observations confirms their very red slopes continue to the infrared, they should be included more quantitatively among the sample.

\section{[Table 4 about here.]}

\section{Discussion}

Fig. 3 plots the orbital elements of the SDSS D-type candidates and the objects observed in this work. The candidates and confirmed D-types display a wide range of orbits throughout the inner belt suggesting these D-types do not all originate from a single source or location such as being disrupted fragments of an originally larger parent body. We note that among the larger, visible-wavelength D-types from Lazzaro et al. (2004) (not plotted), two are located among the Phocaeas and the other three are at the outer edge of the inner belt around 2.45AU.

\section{[Figure 3 about here.]}

Albedos listed in Table 2 are a weighted average from WISE (Mainzer et al. 2011a), IRAS (Tedesco et al. 2002; Ryan and Woodward 2010), and AKARI (Usui et al. 2011) when available or from WISE when that is the only survey with data. The median albedo of our inner belt D-types is 0.09 , with values ranging from 0.07 to 0.11 . This is consistent with albedos found for Bus D-types although slightly higher than the albedos for most Tholen and Bus-DeMeo D-types (Mainzer et al. 2011b).

We estimate there are on the order of 100 inner belt Dtype asteroids with $\mathrm{H}$ magnitudes between 12 and 17 which corresponds to diameters between 2.5 and $20 \mathrm{~km}$. While we do not follow up D-type SDSS candidates in the middle belt, we find 184 candidates. Assuming a similar confirmation rate we expect there to be on the order of 200-250 middle belt Dtypes over the same $\mathrm{H}$ magnitude range. Assuming an average albedo and density we can calculate the total volume and estimated mass of D-type material in the inner belt. For a lower limit we use an albedo of 0.09 , which is the median of the D-types observed in this work and a density of $1.0 \mathrm{~g} / \mathrm{cm}^{3}$. This density agrees with the single D-type density measurement available which is $1.0 \pm 0.02$ for asteroid (624) Hektor (Marchis et al.2013) and is also consistent with densities of comets and transneptunian objects (Carry 2012). We find the lower limit to the total volume and mass to be $2.6 \times 10^{13} \mathrm{~m}^{3}$ and $2.6 \times 10^{16} \mathrm{~kg}$ which represents $\sim 0.01 \%$ of the mass of the inner belt. We calculate an upper limit for the mass assuming a lower albedo $(0.04)$ and a density of $1.8 \mathrm{~g} / \mathrm{cm}^{3}$ which is the at the upper end of the range for C-types (Carry 2012). In this case the mass increases by nearly an order of magnitude. If (908) Buda and the 5 D-types from Lazzaro et al. (2004) are included, which range in diameter from 15 to $30 \mathrm{~km}$, that would increase the mass by another factor of a few assuming the 0.09 albedo and $1.0 \mathrm{~g} / \mathrm{cm}^{3}$ density case. By mass, D-types represent roughly $1,2,15,67 \%$ of the outer belt, Cybeles, Hildas and Trojans (DeMeo and Carry 2013). Inner belt D-types represent, as expected, a very small fraction of the inner belt as well as a small fraction of the total D-type population.

As mentioned in the Results Section, we did not confirm any D-types with $\mathrm{H}>15$. If, in fact, there are fewer D-types at the smallest sizes we sample, that would change the number of Dtypes greater than $2 \mathrm{~km}$ from $\sim 100$ objects to $\sim 25$ (see Table 4), although the total mass would not change substantially.

The currently favored theory of solar system evolution includes periods of planetary migration that displace large numbers of small bodies (e.g. Gomes et al. 2005, Morbidelli et al. 2005; Tsiganis et al. 2005; Walsh et al. 2011). Levison et al. (2009) produced simulations showing that P- and D-type asteroids originating in the outer solar system could have been implanted into the Trojans and could have reached as far as the outer belt. At that time no P- or D-types had been observed in the inner or middle parts of the belt. P-type is a taxonomic class in the Tholen taxonomy (Tholen 1984) with relatively featureless and moderately red spectra and low albedos, that falls within the $\mathrm{X}$-complex in the Bus-DeMeo taxonomy (DeMeo et al. 2009a). Because $\mathrm{C}$ and $\mathrm{P}$ type asteroids are less easily distinguished especially in the near-infrared range we prefer to focus on the more spectrally distinct D-types. We find 3 Dtypes in the inner belt, the largest of which has a diameter of about $10 \mathrm{~km}$ and 1 anomalous D-type (908) Buda with a diameter of about $30 \mathrm{~km}$. The work by Levison et al. (2009) only includes objects with diameters greater than $40 \mathrm{~km}$. Because of the size mismatch between our observations and the simulations, perhaps some of the more numerous smaller bodies reached farther distances inward or these smaller bodies have managed to move from other regions of the belt later on in solar system history. Dynamical models have yet to show how D-types could penetrate into the inner reaches of the Main Belt from the Kuiper Belt under current scenarios of planet formation.

If these objects were originally implanted in the outer belt, or even in the middle belt, the D-type asteroids would still need to cross major resonances, particularly the 3:1. The major resonances have a strong eccentricity pumping effect acting on short (typically $<1 \mathrm{My}$ ) timescales, pushing the objects out of the main belt and into planet crossing space (Gladman et al. 1997). However, there are at least two plausible ways a body could cross. First, a single energetic event such as the break up of a parent body into a family near the 3:1 that could provide enough energy to quickly cross. The varied orbits of the inner belt D-types and lack of evidence for a remnant family on the other side of the 3:1 makes this scenario unlikely.

Alternatively, the Yarkovsky force causes objects with diameters on the order of tens of $\mathrm{km}$ or smaller to drift slowly in semi-major axis space. Nesvorný et al. (2008) investigate the dynamical spread of the Vesta family members over $2 \mathrm{~Gy}$ and found that none of their 132 test objects that entered the $3: 1$ 
resonance succeeded to cross it. In fact, all were removed from the main belt by eccentricity pumping putting them on planetcrossing orbits. Roig et al. (2008a) also examined the possibility of Vesta family members crossing the 3:1 resonance and found that the $\sim 5 \mathrm{~km}$ Vesta-like asteroid (21238) located in the middle belt has only a $\sim 1 \%$ probability of having originated from the Vesta family. They find, however, that objects smaller than $5 \mathrm{~km}$ have a much higher probability of resonance crossing. While many of the inner belt D-types are larger than $5 \mathrm{~km}$, they also have much lower albedos than the Vesta-like objects studied in previous work and are thus generally more affected by Yarkvosky drift (Vokrouhlický and Bottke 2001). Nevertheless, the Yarkovsky effect is dependent on many factors including thermal inertia, shape, density, rotation state and other physical properties. Further work is needed to investigate the efficiency of objects crossing resonances particularly as a function of size and albedo.

In another scenario, these D-types could have been transported during another migration period. Most of the P- and Dtypes were thought to have been transported during a late-stage migration caused by interactions between the giant planets and the Kuiper Belt that destabilized the region and sent bodies inward to the inner solar system (Levison et al.2011). However, in a hypothesized early stage migration, material near and between the giant planets is moved inward when Jupiter migrates inward to about 1.5 AU and then back outward (Walsh et al. 2011). We would expect most of this material to have been $\mathrm{C}$ type, but it is plausible that there was compositional variation among that population that would cause some these bodies to look more like a D-type.

The inner belt D-types have albedos at or above the upper limit of the typical range for D-type asteroids in the outer belt to the Trojans (0.03-0.07, Fernández et al.2003; Mainzer et al. 2011b; DeMeo and Carry 2013). The objects in our sample are much smaller than the typical sizes of D-types measured further out in the solar system. Smaller sizes typically indicate a younger surface age, so it is possible the difference is due to weathering effects. Because of the lack of distinguishing features for D-types, it is also possible that the objects we find in the inner belt are compositionally distinct from other D-types.

Inner belt D-types have a size-frequency distribution drastically different from other regions. All confirmed and candidate D-types in the inner belt have $\mathrm{D} \lesssim 30 \mathrm{~km}$ which have dynamical lifetimes expected to be shorter than the age of the solar system. There are none in the medium $(30-100 \mathrm{~km})$ or large $(>100 \mathrm{~km})$ size range. This means they are most likely collisional fragments of a larger body, however there are no candidate D-type parents in the inner belt. If these D-types originated from another D-type, then the parents are either completely destroyed or were never in the inner belt at all. The presence of these bodies also raise the question as to whether they could originate from a larger parent of a different taxonomic type.

\section{Conclusion}

We identified inner belt D-type candidates from the Sloan Digital Sky Survey Moving Object Catalog and confirmed 3
D-types from new near-infrared spectral observations. We estimate there are $\sim 100$ and $\sim 250$ D-types with diameters between 2.5 and $20 \mathrm{~km}$ in the inner and middle belt, respectively. The average albedo of the inner belt D-types (0.09) is slightly higher than for typical D-types. D-types are thought to originate from the outer solar system, however, models by Levison et al. (2009) show that they are not expected to reach as far as the inner belt. There are many possible scenarios that could explain the presence of these inner belt D-types: i) They were scattered farther than expected during the late-stage migration modeled by Levison et al. (2009). ii) They arrived through another mechanism such as an earlier migration, other planetary scattering, or Yarkovsky drift across the resonances. iii) They are compositionally distinct from other D-types and thus do not require an implantation mechanism.

The sample size in this work is small, but has uncovered an important new population in the inner belt. Future work discovering inner belt D-types with measured visible and nearinfrared spectra and albedos will help us understand the frequency and origin of these bodies.

\section{Acknowledgments}

We thank Bill Bottke for discussions and providing source region probabilities for the NEOs. Observations reported here were obtained at the NASA Infrared Telescope Facility, which is operated by the University of Hawaii under Cooperative Agreement NCC 5-538 with the National Aeronautics and Space Administration, Science Mission Directorate, Planetary Astronomy Program. This paper includes data gathered with the 6.5 meter Magellan Telescopes located at Las Campanas Observatory, Chile. We acknowledge support from the Faculty of the European Space Astronomy Centre (ESAC) for FD's visit. DP is grateful to the AXA research fund. This material is based upon work supported by the National Aeronautics and Space Administration under Grant No. NNX12AL26G issued through the Planetary Astronomy Program and by the National Science Foundation under Grant 0907766. Any opinions, findings, and conclusions or recommendations expressed in this article are those of the authors and do not necessarily reflect the views of the National Aeronautics and Space Administration or the National Science Foundation. 


\section{References}

Alvarez-Candal, A., Fornasier, S., Barucci, M. A., de Bergh, C., Merlin, F., 2008. Visible spectroscopy of the new ESO large program on transNeptunian objects and Centaurs. Part 1. Astronomy and Astrophysics 487, 741-748.

Binzel, R. P., Rivkin, A. S., Stuart, J. S., Harris, A. W., Bus, S. J., Burbine, T. H., 2004. Observed spectral properties of near-Earth objects: results for population distribution, source regions, and space weathering processes. Icarus 170, 259-294.

Binzel, R. P., Thomas, C. A., DeMeo, F. E., Tokunaga, A., Rivkin, A. S., Bus, S. J., 2006. The MIT-Hawaii-IRTF Joint Campaign for NEO Spectral Reconnaissance. In: Mackwell, S., Stansbery, E. (Eds.), 37th Annual Lunar and Planetary Science Conference. Vol. 37 of Lunar and Planetary Inst. Technical Report. p. 1491.

Bottke, W. F., Morbidelli, A., Jedicke, R., Petit, J.-M., Levison, H. F., Michel, P., Metcalfe, T. S., 2002. Debiased Orbital and Absolute Magnitude Distribution of the Near-Earth Objects. Icarus 156, 399-433.

Bus, S. J., 1999. Compositional structure in the asteroid belt: Results of a spectroscopic survey. Ph.D. thesis, MASSACHUSETTS INSTITUTE OF TECHNOLOGY

Bus, S. J., Binzel, R. P., 2002a. Phase II of the Small Main-Belt Asteroid Spectroscopic Survey, A Feature-Based Taxonomy. Icarus 158, 146-177.

Bus, S. J., Binzel, R. P., 2002b. Phase II of the Small Main-Belt Asteroid Spectroscopic Survey, The Observations. Icarus 158, 106-145

Carry, B., 2012. Density of asteroids. Planetary and Space Science 73, 98-118

Carvano, J. M., Hasselmann, P. H., Lazzaro, D., Mothé-Diniz, T., 2010. SDSSbased taxonomic classification and orbital distribution of main belt asteroids. Astron. Astrophysics 510, A43+.

Cruikshank, D. P., Kerridge, J. F., 1992. Organic material: Asteroids, meteorites, and planetary satellites. NASA STI/Recon Technical Report N 93, 18555.

Cruikshank, D. P., Roush, T. L., Bartholomew, M. J., Geballe, T. R., Pendleton, Y. J., White, S. M., Bell, J. F., Davies, J. K., Owen, T. C., de Bergh, C., Tholen, D. J., Bernstein, M. P., Brown, R. H., Tryka, K. A., Dalle Ore, C. M., 1998. The Composition of Centaur 5145 Pholus. Icarus 135, 389407.

Cushing, M. C., Vacca, W. D., Rayner, J. T., 2004. Spextool: A Spectral Extraction Package for SpeX, a 0.8-5.5 Micron Cross-Dispersed Spectrograph. PASP 116, 362-376.

DeMeo, F., Binzel, R. P., 2008. Comets in the near-Earth object population. Icarus 194, 436-449.

DeMeo, F. E., Binzel, R. P., Slivan, S. M., Bus, S. J., 2009a. An extension of the Bus asteroid taxonomy into the near-infrared. Icarus 202, 160-180.

DeMeo, F. E., Carry, B., 2013. The taxonomic distribution of asteroids from multi-filter all-sky photometric surveys. Icarus 226, 723-741.

DeMeo, F. E., Fornasier, S., Barucci, M. A., Perna, D., Protopapa, S., AlvarezCandal, A., Delsanti, A., Doressoundiram, A., Merlin, F., de Bergh, C., 2009b. Visible and near-infrared colors of Transneptunian objects and Centaurs from the second ESO large program. Astronomy and Astrophysics 493, $283-290$.

Dotto, E., Fornasier, S., Barucci, M. A., Licandro, J., Boehnhardt, H., Hainaut, O., Marzari, F., de Bergh, C., De Luise, F., 2006. The surface composition of Jupiter Trojans: Visible and near-infrared survey of dynamical families. Icarus 183, 420-434.

Emery, J. P., Burr, D. M., Cruikshank, D. P., 2011. Near-infrared Spectroscopy of Trojan Asteroids: Evidence for Two Compositional Groups. Astronomical Journal 141, 25.

Fernández, Y. R., Sheppard, S. S., Jewitt, D. C., 2003. The Albedo Distribution of Jovian Trojan Asteroids. Astronomical Journal 126, 1563-1574.

Fitzsimmons, A., Dahlgren, M., Lagerkvist, C.-I., Magnusson, P., Williams, I. P., 1994. A spectroscopic survey of D-type asteroids. Astronomy and Astrophysics 282, 634-642.

Fornasier, S., Barucci, M. A., de Bergh, C., Alvarez-Candal, A., DeMeo, F., Merlin, F., Perna, D., Guilbert, A., Delsanti, A., Dotto, E., Doressoundiram, A., 2009. Visible spectroscopy of the new ESO large programme on transNeptunian objects and Centaurs: final results. Astronomy and Astrophysics 508, 457-465

Fornasier, S., Clark, B. E., Dotto, E., Migliorini, A., Ockert-Bell, M., Barucci, M. A., 2010. Spectroscopic survey of M-type asteroids. Icarus 210, 655673.
Fornasier, S., Dotto, E., Hainaut, O., Marzari, F., Boehnhardt, H., De Luise, F., Barucci, M. A., 2007. Visible spectroscopic and photometric survey of Jupiter Trojans: Final results on dynamical families. Icarus 190, 622-642.

Fornasier, S., Dotto, E., Marzari, F., Barucci, M. A., Boehnhardt, H., Hainaut, O., de Bergh, C., 2004. Visible spectroscopic and photometric survey of L5 Trojans: investigation of dynamical families. Icarus 172, 221-232.

Gladman, B. J., Migliorini, F., Morbidelli, A., Zappala, V., Michel, P., Cellino, A., Froeschle, C., Levison, H. F., Bailey, M., Duncan, M., 1997. Dynamical lifetimes of objects injected into asteroid belt resonances. Science 277, 197201.

Gomes, R., Levison, H. F., Tsiganis, K., Morbidelli, A., 2005. Origin of the cataclysmic Late Heavy Bombardment period of the terrestrial planets. Nature 435, 466-469.

Gradie, J., Tedesco, E., 1982. Compositional structure of the asteroid belt. Science $216,1405-1407$.

Gradie, J., Veverka, J., 1980. The composition of the Trojan asteroids. Nature $283,840-842$.

Grav, T., Mainzer, A. K., Bauer, J. M., Masiero, J. R., Nugent, C. R., 2012. WISE/NEOWISE Observations of the Jovian Trojan Population: Taxonomy. Astrophysical Journal 759, 49

Ivezić, Ž., Lupton, R. H., Jurić, M., Tabachnik, S., Quinn, T., Gunn, J. E., Knapp, G. R., Rockosi, C. M., Brinkmann, J., 2002. Color Confirmation of Asteroid Families. Astronomical Journal 124, 2943-2948.

Ivezić, Ž., Tabachnik, S., Rafikov, R., Lupton, R. H., Quinn, T., Hammergren, M., Eyer, L., Chu, J., Armstrong, J. C., Fan, X., Finlator, K., Geballe, T. R., Gunn, J. E., Hennessy, G. S., Knapp, G. R., Leggett, S. K., Munn, J. A., Pier, J. R., Rockosi, C. M., Schneider, D. P., Strauss, M. A., Yanny, B., Brinkmann, J., Csabai, I., Hindsley, R. B., Kent, S., Lamb, D. Q., Margon, B., McKay, T. A., Smith, J. A., Waddel, P., York, D. G., the SDSS Collaboration, 2001. Solar System Objects Observed in the Sloan Digital Sky Survey Commissioning Data. Astronomical Journal 122, 2749-2784.

Lagerkvist, C.-I., Fitzsimmons, A., Magnusson, P., Williams, I. P., 1993. Investigations of D-type asteroids. Monthly Notices of the Royal Astronomical Society 260,679

Lazzaro, D., Angeli, C. A., Carvano, J. M., Mothé-Diniz, T., Duffard, R., Florczak, M., 2004. $\mathrm{S}^{3} \mathrm{OS}^{2}$ : the visible spectroscopic survey of 820 asteroids. Icarus 172, 179-220.

Levison, H. F., Bottke, W. F., Gounelle, M., Morbidelli, A., Nesvorný, D., Tsiganis, K., 2009. Contamination of the asteroid belt by primordial transNeptunian objects. Nature 460, 364-366.

Levison, H. F., Morbidelli, A., Tsiganis, K., Nesvorný, D., Gomes, R., 2011. Late orbital instabilities in the outer planets induced by interaction with selfgravitating planetesimal disk. Astronomical Journal 142, 142-152.

Lord, S. D., 1992. A new software tool for computing earth's atmospheric transmission of near- and far-infrared radiation. NASA Tech. Mem. (103957).

Mainzer, A., Bauer, J., Grav, T., Masiero, J., Cutri, R. M., Dailey, J., Eisenhardt, P., McMillan, R. S., Wright, E., Walker, R., Jedicke, R., Spahr, T., Tholen, D., Alles, R., Beck, R., Brandenburg, H., Conrow, T., Evans, T., Fowler, J., Jarrett, T., Marsh, K., Masci, F., McCallon, H., Wheelock, S., Wittman, M., Wyatt, P., DeBaun, E., Elliott, G., Elsbury, D., Gautier, IV, T., Gomillion, S., Leisawitz, D., Maleszewski, C., Micheli, M., Wilkins, A., 2011a. Preliminary Results from NEOWISE: An Enhancement to the Wide-field Infrared Survey Explorer for Solar System Science. Astrophysical Journal 731, 53.

Mainzer, A., Grav, T., Masiero, J., Hand, E., Bauer, J., Tholen, D., McMillan, R. S., Spahr, T., Cutri, R. M., Wright, E., Watkins, J., Mo, W., Maleszewski, C., 2011b. NEOWISE Studies of Spectrophotometrically Classified Asteroids: Preliminary Results. Astrophysical Journal 741, 90.

Marchis, F., Durech, J., Castillo-Rogez, J., Cuk, M., Berthier, J., Wong, H. M. Kalas, P., Duchene, G., van Dam, M., 2013. Density and interior of binary jovian-trojan asteroids: The (624) hektor case. EPSC Abstracts 8 (849).

Masiero, J. R., Mainzer, A. K., Grav, T., Bauer, J. M., Cutri, R. M., Dailey, J., Eisenhardt, P. R. M., McMillan, R. S., Spahr, T. B., Skrutskie, M. F., Tholen, D., Walker, R. G., Wright, E. L., DeBaun, E., Elsbury, D., Gautier, IV, T., Gomillion, S., Wilkins, A., 2011. Main Belt Asteroids with WISE/NEOWISE. I. Preliminary Albedos and Diameters. Astrophysical Journal 741, 68.

Morbidelli, A., Levison, H. F., Tsiganis, K., Gomes, R., 2005. Chaotic capture of Jupiter's Trojan asteroids in the early Solar System. Nature 435, 462-465.

Moskovitz, N. A., Jedicke, R., Gaidos, E., Willman, M., Nesvorný, D., Fevig, R., Ivezić, Ž., 2008. The distribution of basaltic asteroids in the Main Belt. Icarus 198, 77-90. 
Nesvorný, D., Roig, F., Gladman, B., Lazzaro, D., Carruba, V., Mothé-Diniz, T., 2008. Fugitives from the Vesta family. Icarus 193, 85-95.

Ockert-Bell, M. E., Clark, B. E., Shepard, M. K., Rivkin, A. S., Binzel, R. P., Thomas, C. A., DeMeo, F. E., Bus, S. J., Shah, S., 2008. Observations of $\mathrm{X} / \mathrm{M}$ asteroids across multiple wavelengths. Icarus 195, 206-219.

Perna, D., Barucci, M. A., Fornasier, S., DeMeo, F. E., Alvarez-Candal, A., Merlin, F., Dotto, E., Doressoundiram, A., de Bergh, C., 2010. Colors and taxonomy of Centaurs and trans-Neptunian objects. Astronomy and Astrophysics 510, A53.

Rayner, J. T., Toomey, D. W., Onaka, P. M., Denault, A. J., Stahlberger, W. E., Vacca, W. E., Cushing, M. C., Wang, S., 2003. Spex: A medium-resolution 0.8-5.5 micron spectrograph and imager for the nasa infrared telescope facility. Astron. Soc. of the Pacific 115, 362-382.

Rivkin, A. S., Binzel, R. P., Sunshine, J., Bus, S. J., Burbine, T. H., Saxena, A., 2004. Infrared spectroscopic observations of 69230 Hermes (1937 UB): possible unweathered endmember among ordinary chondrite analogs. Icarus $172,408-414$

Roig, F., Nesvorný, D., Gil-Hutton, R., Lazzaro, D., 2008a. V-type asteroids in the middle main belt. Icarus 194, 125-136.

Roig, F., Ribeiro, A. O., Gil-Hutton, R., 2008b. Taxonomy of asteroid families among the Jupiter Trojans: comparison between spectroscopic data and the Sloan Digital Sky Survey colors. Astron. Astrophysics 483, 911-931.

Ryan, E. L., Woodward, C. E., 2010. Rectified Asteroid Albedos and Diameters from IRAS and MSX Photometry Catalogs. Astronomical Journal 140, 933 943.

Simcoe, R. A., Burgasser, A. J., Schechter, P. L., Fishner, J., Bernstein, R. A., Bigelow, B. C., Pipher, J. L., Forrest, W., McMurtry, C., Smith, M. J., Bochanski, J. J., 2013. FIRE: A Facility Class Near-Infrared Echelle Spectrometer for the Magellan Telescopes. PASP 125, 270-286.

Tedesco, E. F., Noah, P. V., Noah, M. C., Price, S. D., 2002. The Supplemental IRAS Minor Planet Survey. Astronomical Journal 123, 1056-1085.

Tholen, D. J., 1984. Asteroid taxonomy from cluster analysis of photometry. Ph.D. thesis, University of Arizona.

Tody, D., 1993. Iraf in the nineties. in astronomical data. In Astronomical Data Analysis Software and Systems II.

Tsiganis, K., Gomes, R., Morbidelli, A., Levison, H. F., 2005. Origin of the orbital architecture of the giant planets of the Solar System. Nature 435, 459-461.

Usui, F., Kuroda, D., Müller, T. G., Hasegawa, S., Ishiguro, M., Ootsubo, T., Ishihara, D., Kataza, H., Takita, S., Oyabu, S., Ueno, M., Matsuhara, H., Onaka, T., Jun 2011. Asteroid Catalog Using Akari: AKARI/IRC MidInfrared Asteroid Survey. Publications of the Astronomical Society of Japan 63, 1117-1138.

Vokrouhlický, D., Bottke, Jr., W. F., 2001. The Yarkovsky thermal force on small asteroids and their fragments. Choosing the right albedo. Astronomy and Astrophysics 371, 350-353.

Walsh, K. J., Morbidelli, A., Raymond, S. N., O’Brien, D. P., Mandell, A. M., 2011. A low mass for Mars from Jupiter's early gas-driven migration. Nature 475, 206-209.

Yang, B., Jewitt, D., 2011. A Near-infrared Search for Silicates in Jovian Trojan Asteroids. Astronomical Journal 141, 95. 


\section{List of Figures}

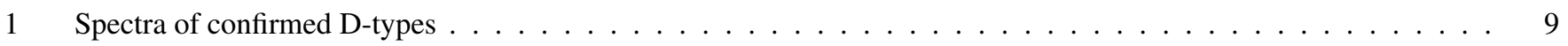

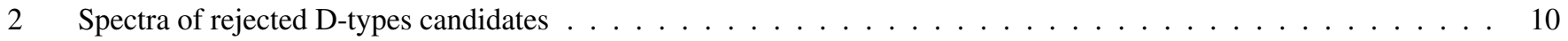

3 Orbital distribution of D-types in the inner belt $\ldots \ldots \ldots \ldots$ 


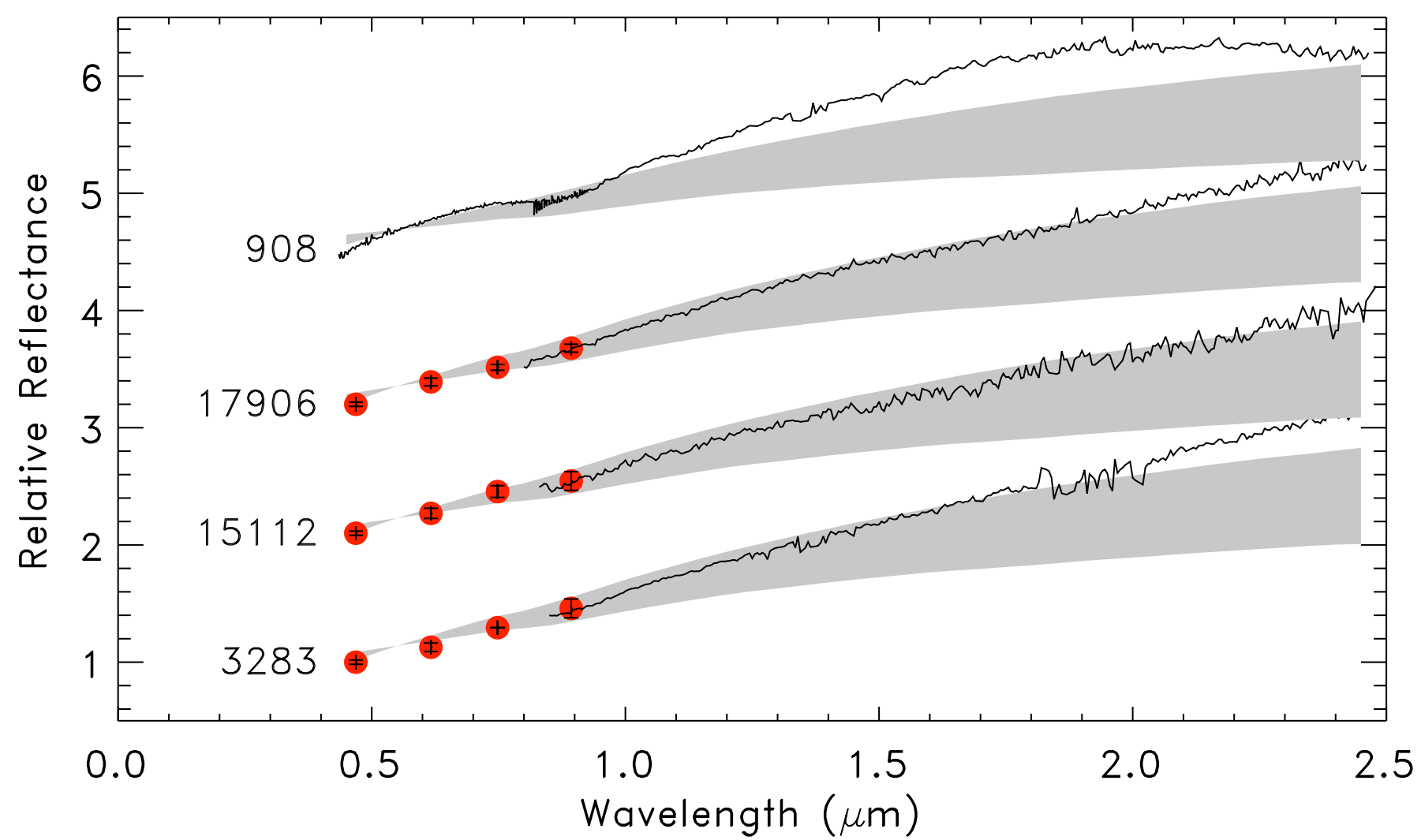

Figure 1: Plot of confirmed D-types based on near-infrared spectral measurements from this work for three objects plus asteroid (908) from (DeMeo et al. 2009a). SDSS colors are plotted as red dots with black error bars (smaller than the size of the dot). The spectra are plotted in black. The gray region bounds plus and minus one sigma from the mean of the D-type class. The three SDSS D-types have nearly identical spectra. 


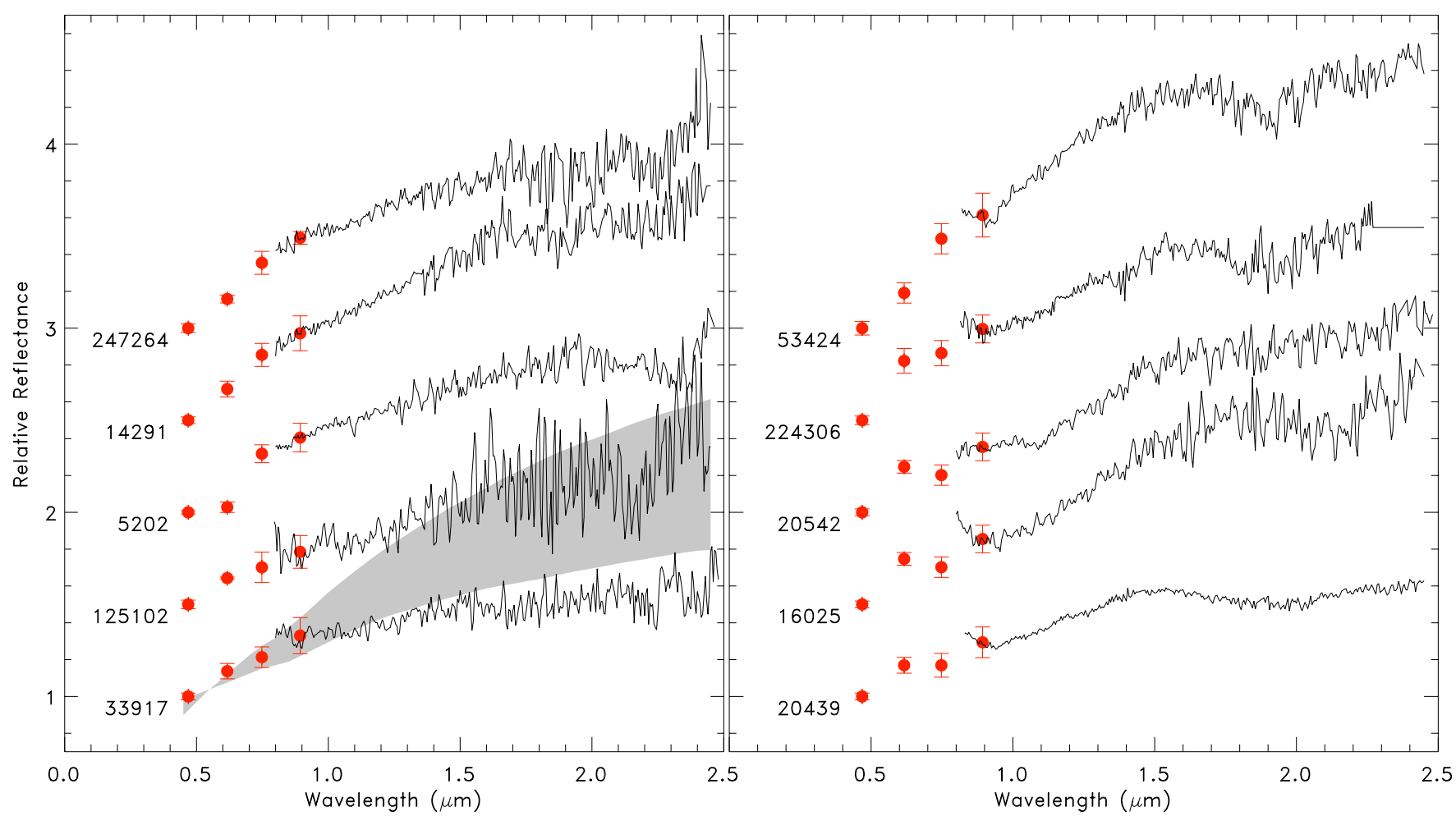

Figure 2: Plot of SDSS candidates that are not D-types based on near-infrared follow up observations. SDSS colors are plotted as red dots with red error bars. The spectra are plotted in black. The gray region plotted with the first spectrum shows the boundaries plus and minus one sigma from the mean of the D-type class illustrating the difference between D-types and these spectra. On the left are featureless or subtly featured spectra with slopes that place them in the C or X complex. On the right are spectra with 1 and $2 \mu \mathrm{m}$ features that place them in the $\mathrm{S}, \mathrm{K}$, or L classes. 


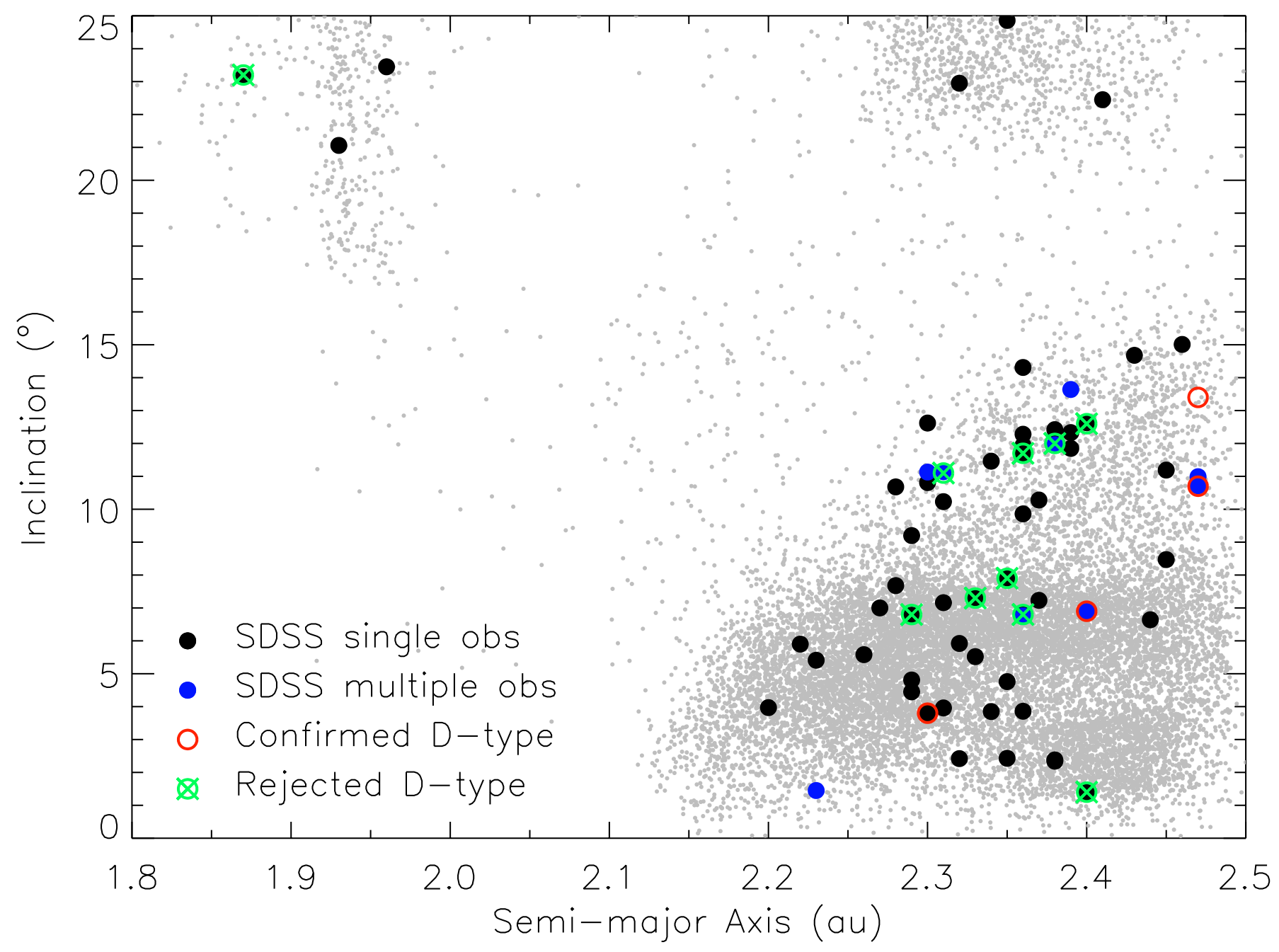

Figure 3: Orbital distribution of D-types in the inner belt. This plot shows the location of the candidate D-types located closer than 2.5AU. The bottom right region is the inner main belt. The top left is the Hungarias and the top right is the Phocaeas. A sample of MBAs are plotted in gray to illustrate the structure of the region. SDSS candidates are plotted as black or blue dots for objects with single or multiple observations respectively. Red circles indicate D-types that have been confirmed with near-infrared data. Green X marks objects with follow up observations that do not classify as D-types. 


\section{List of Tables}

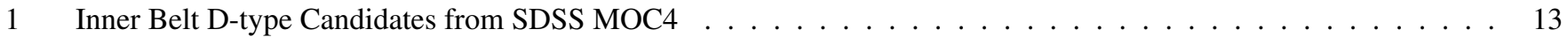

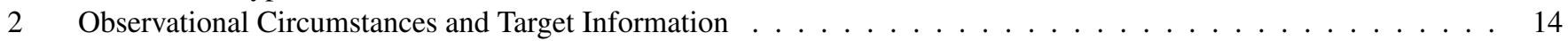

3 Source Region Probabilities for D-type NEOs . . . . . . . . . . . . . . . . . . . . . . . . . . . . 15

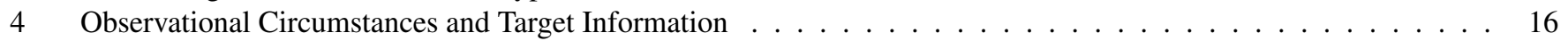


Table 1: Inner Belt D-type Candidates from SDSS MOC4

\begin{tabular}{|c|c|c|c|c|c|}
\hline \multicolumn{2}{|c|}{ Designation } & \multirow{2}{*}{$\begin{array}{c}\mathrm{H} \\
(\mathrm{mag})\end{array}$} & \multirow{2}{*}{$\begin{array}{c}\mathrm{a} \\
(\mathrm{au})\end{array}$} & \multirow[t]{2}{*}{$\bar{e}$} & \multirow{2}{*}{$\begin{array}{r}\mathrm{i} \\
\left({ }^{\circ}\right)\end{array}$} \\
\hline$(\#)^{1}$ & (Name) & & & & \\
\hline \multicolumn{6}{|c|}{ Inner belt candidates } \\
\hline 2806 & Graz & 13.1 & 2.38 & 0.05 & 2.3 \\
\hline $3283 *$ & Skorina & 12.6 & 2.40 & 0.10 & 6.9 \\
\hline 5202 & $1983 \mathrm{XX}$ & 13.0 & 2.40 & 0.17 & 12.6 \\
\hline 5302 & Romanoserr & 13.9 & 2.33 & 0.04 & 2.1 \\
\hline 8069 & Benweiss & 14.0 & 2.34 & 0.07 & 6.1 \\
\hline 8856 & Celastrus & 14.3 & 2.35 & 0.10 & 2.4 \\
\hline 10573 & Piani & 14.5 & 2.45 & 0.16 & 14.8 \\
\hline 14291 & $1104 \mathrm{~T}-1$ & 13.9 & 2.35 & 0.13 & 11.7 \\
\hline $15112 *$ & Arlenewolf & 14.2 & 2.30 & 0.15 & 3.8 \\
\hline 16025 & 1999 CA104 & 14.1 & 2.29 & 0.21 & 6.8 \\
\hline $17906^{*}$ & 1999 FG32 & 13.5 & 2.47 & 0.17 & 10.7 \\
\hline 20180 & Annakoleny & 14.5 & 2.48 & 0.12 & 12.9 \\
\hline 20243 & 1998 DB36 & 13.9 & 2.34 & 0.22 & 11.5 \\
\hline 20439 & 1999 JM28 & 13.9 & 2.40 & 0.19 & 1.4 \\
\hline 20452 & 1999 KG4 & 14.0 & 2.35 & 0.24 & 7.9 \\
\hline 20749 & 2000 AD199 & 13.7 & 2.45 & 0.15 & 14.3 \\
\hline 21606 & 1999 FH6 & 15.0 & 2.18 & 0.07 & 0.9 \\
\hline 22788 & von Steube & 13.7 & 2.45 & 0.21 & 8.5 \\
\hline 25718 & 2000 АH 170 & 14.7 & 2.29 & 0.11 & 10.9 \\
\hline 27435 & 2000 FZ35 & 14.4 & 2.47 & 0.12 & 12.8 \\
\hline 27842 & 1994 QJ & 14.2 & 2.35 & 0.23 & 24.9 \\
\hline 29452 & 1997 RV2 & 14.4 & 2.39 & & 12.3 \\
\hline 33917 & 2000 LK19 & 14.9 & 2.33 & 0.11 & 7.3 \\
\hline 33964 & 2000 NS10 & 14.9 & 2.36 & & 6.8 \\
\hline 34692 & 2001 KE61 & 15.0 & 2.31 & 0.25 & 7.2 \\
\hline 35058 & 1985 RP4 & 15.0 & & & 3.8 \\
\hline 38699 & 2000 QX63 & 15.7 & 2.27 & 0.09 & 7.0 \\
\hline 39809 & Fukuchan & 16.2 & 2.23 & 0.22 & 5.4 \\
\hline 39881 & 1998 EK11 & 14.7 & 2.36 & 0.19 & 3.9 \\
\hline 44169 & 1998 KK2 & 14.0 & 2.46 & 0.28 & 14.7 \\
\hline 47320 & 1999 XA15 & 13.7 & 2.41 & 0.09 & 22.4 \\
\hline 48049 & 2001 DB90 & 15.1 & 2.47 & 0.13 & 11.0 \\
\hline 48763 & $1997 \mathrm{JZ}$ & 15.0 & 2.39 & 0.13 & 13.6 \\
\hline 49092 & 1998 RK71 & 15.1 & 2.23 & 0.06 & 6.1 \\
\hline 52570 & 1997 JC1 & 14.7 & 2.38 & 0.13 & 13.8 \\
\hline 55391 & 2001 ST277 & 14.6 & 2.39 & 0.20 & 11.8 \\
\hline 55567 & 2002 CS6 & 13.5 & 2.32 & 0.27 & 22.9 \\
\hline 55590 & 2002 PB97 & 15.3 & 2.34 & 0.25 & 3.9 \\
\hline 57546 & 2001 TO21 & 15.7 & 2.44 & 0.09 & 6.6 \\
\hline 58005 & 2002 TR207 & 15.5 & 2.37 & 0.07 & 10.3 \\
\hline 58684 & 1998 AA11 & 15.8 & 2.42 & 0.16 & 3.5 \\
\hline 65915 & 1998 FO34 & 14.2 & 2.34 & 0.09 & 12.9 \\
\hline 68004 & 2000 XD38 & 15.2 & 2.44 & 0.11 & 14.5 \\
\hline 68448 & Sidneywolf & 15.3 & 2.29 & 0.24 & 9.2 \\
\hline 73598 & $2912 \mathrm{~T}-2$ & 17.0 & 2.23 & 0.14 & 1.5 \\
\hline 76973 & 2001 ВТ53 & 15.0 & 2.36 & 0.15 & 11.9 \\
\hline 80532 & 2000 AV71 & 15.9 & 2.29 & 0.04 & 4.5 \\
\hline 84480 & 2002 ТМ266 & 14.8 & 2.30 & 0.26 & 11.1 \\
\hline 84802 & 2002 YC1 & 14.8 & 2.36 & 0.22 & 9.9 \\
\hline 85054 & 6841 P-L & 16.4 & 2.31 & 0.12 & 4.0 \\
\hline 96199 & 1992 EY24 & 15.8 & 2.20 & 0.12 & 4.0 \\
\hline 97965 & 2000 QW143 & 15.7 & 2.36 & 0.14 & 14.3 \\
\hline 99449 & 2002 CJ30 & $1 B 6.4$ & 2.29 & 0.11 & 4.8 \\
\hline 104163 & 2000 EL76 & 15.9 & 2.36 & 0.11 & 12.3 \\
\hline 109253 & 2001 QT103 & 16.0 & 2.48 & 0.22 & 14.9 \\
\hline 111899 & 2002 FD11 & 16.5 & 2.21 & 0.07 & 4.0 \\
\hline 122596 & 2000 RG35 & 14.5 & 2.43 & 0.11 & 14.7 \\
\hline 123240 & 2000 UU59 & 15.8 & 2.32 & 0.25 & 5.9 \\
\hline
\end{tabular}


Table 2: Observational Circumstances and Target Information

\begin{tabular}{|c|c|c|c|c|c|c|c|c|c|c|c|}
\hline \multicolumn{2}{|c|}{ Designation } & \multirow{2}{*}{$\begin{array}{c}\text { Phase } \\
\left({ }^{\circ}\right)\end{array}$} & \multirow{2}{*}{$\begin{array}{c}\mathrm{V} \\
(\mathrm{mag})\end{array}$} & \multirow[t]{2}{*}{ Class } & \multirow{2}{*}{$\begin{array}{c}\text { Slope }^{a} \\
(\% / 1000 A)\end{array}$} & \multirow{2}{*}{ Albedq $^{b}$} & \multirow{2}{*}{$\begin{array}{c}\text { Est. D } \\
(\mathrm{km})\end{array}$} & \multirow{2}{*}{$\begin{array}{c}\mathrm{H} \\
(\mathrm{mag})\end{array}$} & \multirow{2}{*}{$\begin{array}{c}\text { SDSS } \\
(\text { Class })^{c}\end{array}$} & \multicolumn{2}{|c|}{ New Observations } \\
\hline (\#) & (Name) & & & & & & & & & (Tel.) & \\
\hline 908 & Buda & 13.9 & 15.2 & $\mathrm{D}$ & $6.51 \pm 0.13$ & $0.114 \pm 0.025$ & 29 & 10.7 & - & & 2006 \\
\hline 3283 & Skorina & 5.1 & 15.2 & $\mathrm{D}$ & $6.39 \pm 0.03$ & $0.094 \pm 0.021$ & 13 & 12.6 & $\mathrm{D}, \mathrm{D}, \mathrm{X}, \mathrm{C}$ & IRTF & $2012 / 0$ \\
\hline 15112 & Arlenewolfe & 10.5 & 18.1 & $\mathrm{D}$ & $5.59 \pm 0.04$ & $0.076 \pm 0.002$ & 7 & 14.2 & $\mathrm{D}$ & IRTF & $2012 / 1$ \\
\hline 17906 & 1999 FG32 & 19.9 & 16.6 & $\mathrm{D}$ & $5.89 \pm 0.03$ & $0.072 \pm 0.009$ & 10 & 13.5 & $\mathrm{D}, \mathrm{D}$ & IRTF & 2012 \\
\hline 5202 & $1983 X X$ & 20.1 & 17.1 & $\mathrm{C} / \mathrm{X}$ & $1.87 \pm 0.07$ & $0.091 \pm 0.002$ & 11 & 13.0 & $\mathrm{D}$ & IRTF & 2012 \\
\hline 14291 & $1104 \mathrm{~T}-1$ & 16.4 & 17.5 & $\mathrm{X} / \mathrm{D}$ & $2.98 \pm 0.07$ & $0.102 \pm 0.018$ & 7 & 13.9 & $\mathrm{D}$ & IRTF & $2012 / 0$ \\
\hline 16025 & 1999 CA104 & 10.4 & 18 & $\mathrm{~S}$ & $3.71 \pm 0.10$ & $0.159 \pm 0.011$ & 5 & 14.1 & $\mathrm{D}$ & IRTF & 2013 \\
\hline 20439 & 1999 JM28 & 1.7 & 15.9 & S & $1.29 \pm 0.05$ & $0.331 \pm 0.015$ & 4 & 13.9 & $\mathrm{D}$ & IRTF & 2013 \\
\hline 20452 & 1999 KG4 & 20.4 & 19.3 & $\mathrm{~K}$ & $3.49 \pm 0.08$ & $0.220 \pm 0.039$ & 4 & 14.0 & $\mathrm{D}$ & IRTF & 2012 \\
\hline 33917 & 2000 LK19 & 3.6 & 17.9 & $\mathrm{C} / \mathrm{X}$ & $1.18 \pm 0.06$ & $0.114 \pm 0.015$ & 4 & 14.9 & $\mathrm{D}$ & IRTF & 2013 \\
\hline 53424 & 1999 SC3 & 24.1 & 17.9 & $\mathrm{~S} / \mathrm{L}$ & $2.62 \pm 0.08$ & - & - & 15.2 & $\mathrm{D}$ & IRTF & 2012 \\
\hline 125102 & $2001 \mathrm{UH} 35$ & 23.3 & 19.1 & $\mathrm{X} / \mathrm{D}$ & $2.87 \pm 0.15$ & - & - & 15.3 & $\mathrm{D}, \mathrm{D}, \mathrm{K}$ & IRTF & 2013 \\
\hline 224306 & 2005 UV8 & 8.6 & 18.3 & $S$ & $2.18 \pm 0.07$ & - & - & 16.1 & $\mathrm{D}, \mathrm{D}$ & Magellan & $2012 / 0$ \\
\hline 247264 & 2001 SW8 & 8.1 & 18.1 & $\mathrm{C} / \mathrm{X}$ & $2.17 \pm 0.08$ & $0.065 \pm 0.024$ & - & 16.3 & $\mathrm{D}, \mathrm{D}$ & IRTF, Magellan & $2012 / 0$ \\
\hline
\end{tabular}

${ }^{a}$ Errors include only the formal error in the slope calculation.

${ }^{b}$ Reported albedos are either from WISE (Mainzer et al. 2011a Masiero et al. 2011) or are weighted averages from WISE, IRAS Tedesco et al. 2002. Ryan and Woodward 2010), and AKARI (Usui et al.2011) when multiple measurements are available.

${ }^{c}$ One class is listed for each SDSS observation. 
Table 3: Source Region Probabilities for D-type NEOs

\begin{tabular}{lcccccccccc}
\hline Asteroid & Orbi $^{\mid}$ & $\mathrm{a}(\mathrm{AU})$ & $\mathrm{e}$ & $\mathrm{i}(\mathrm{deg})$ & $\mathrm{T}_{J}$ & $\mathrm{P}_{J F C}$ & $\mathrm{P}_{O B}$ & $\mathrm{P}_{3: 1}$ & $\mathrm{P}_{M C}$ & $\mathrm{P}_{v_{6}}$ \\
\hline 52762 & APO & 2.419 & 0.651 & 33.9 & 3.01 & 0.000 & 0.034 & 0.734 & 0.159 & 0.072 \\
162998 & AMO & 1.926 & 0.474 & 1.7 & 3.77 & 0.000 & 0.095 & 0.053 & 0.202 & 0.650 \\
170891 & AMO & 1.983 & 0.405 & 8.1 & 3.74 & 0.000 & 0.000 & 0.032 & 0.299 & 0.670 \\
2003 UC20 & ATE & 0.781 & 0.337 & 3.8 & 7.39 & 0.000 & 0.000 & 0.145 & 0.332 & 0.523 \\
2005 DD & APO & 1.933 & 0.568 & 7.3 & 3.69 & 0.000 & 0.000 & 0.073 & 0.324 & 0.603 \\
2006 MJ10 & APO & 1.876 & 0.586 & 39.3 & 3.53 & 0.000 & 0.000 & 0.331 & 0.290 & 0.379 \\
2013 AH11 & AMO & 2.274 & 0.521 & 28.3 & 3.28 & 0.000 & 0.000 & 0.856 & 0.055 & 0.089 \\
& & & & & & & & & & \\
3552 & AMO & 4.222 & 0.713 & 31.0 & 2.31 & 1.000 & 0.000 & 0.000 & 0.000 & 0.000 \\
17274 & AMO & 2.725 & 0.558 & 5.6 & 3.10 & 0.028 & 0.750 & 0.071 & 0.144 & 0.008 \\
326732 & AMO & 2.705 & 0.575 & 6.3 & 3.10 & 0.028 & 0.750 & 0.071 & 0.144 & 0.008 \\
2000 PG3 & APO & 2.824 & 0.856 & 22.0 & 2.55 & 0.929 & 0.025 & 0.037 & 0.002 & 0.007 \\
2011 BE38 & APO & 2.620 & 0.719 & 7.9 & 2.96 & 0.438 & 0.346 & 0.128 & 0.064 & 0.025 \\
\hline
\end{tabular}

${ }^{a} \mathrm{NEO}$ orbits are labeled as Amor (AMO), Apollo (APO) and Aten (ATE) 
Table 4: Observational Circumstances and Target Information

\begin{tabular}{llllll}
\hline $\mathbf{H}$ & $\mathbf{N}_{\text {SDSS,D }}{ }^{a}$ & $\left.\mathbf{N}_{\text {SDSS, tota }}\right]^{b}$ & $\mathbf{D ~ f r a c t i o n}^{c}$ & $\mathbf{N}_{\text {MPd }}{ }^{d}$ & $\mathbf{N}_{\text {MPC,D }}{ }^{e}$ \\
\hline $12-13$ & 0.4 & 161 & 0.00248 & 712 & $1.8_{-1.6}^{+8.7}$ \\
$13-14$ & 2.3 & 847 & 0.00248 & 3185 & $8.6_{-6.5}^{+31.6}$ \\
$14-15$ & 4.1 & 2209 & 0.00167 & 10236 & $19.0_{-10.9}^{+57.0}$ \\
$15-16$ & 4.5 & 3860 & 0.00106 & 24928 & $29.1_{-16.7}^{+87.2}$ \\
$16-17$ & 3.5 & 3635 & 0.00085 & 69368 & $66.8_{-42.1}^{+215.7}$ \\
Total: & & 10712 & & 108429 & 125.3 \\
\hline
\end{tabular}

${ }^{a}$ The number of inner belt D-types expected statistically in the SDSS sample calculated as the detection rate times the number of D-type candidates from the SDSS sample.

${ }^{b}$ The total number of objects in the inner belt observed in our SDSS sample.

${ }^{c}$ The fraction of D-types at each $\mathrm{H}$ magnitude range calculated as $\mathrm{N}_{S D S S, D} / \mathrm{N}_{\text {SDSS,total }}$

${ }^{d}$ The total number of inner belt asteroids at each $\mathrm{H}$ magnitude range from the MPC with a correction for incompleteness in the last bin.

${ }^{e}$ The total number of inner belt D-types expected statistically. The error bars are based on the Poisson upper and lower limits as described in the text. 